\title{
Study on the Compound Path of Living Environment Renovation Under the Background of "Renovation and Restoration" to Old Communities
}

\author{
Dong Rui \\ School of Culture Communication \& Design, Zhejiang University of Finance \& Economics Dongfang College, Hangzhou, China \\ Email address: \\ dongxiaoyaya@126.com \\ To cite this article: \\ Dong Rui. Study on the Compound Path of Living Environment Renovation Under the Background of "Renovation and Restoration" to Old \\ Communities. Urban and Regional Planning. Vol. 5, No. 2, 2020, pp. 40-49. doi: 10.11648/j.urp.20200502.12
}

Received: May 16, 2020; Accepted: May 25, 2020; Published: June 3, 2020

\begin{abstract}
As a product of a certain era, the old community is now faced with many serious problems that cannot be completely changed, such as the simple functional structure of space, lacking of community vitality, losing of cultural characteristics and the rupture of historical context. Which make it difficult to adapt to diversified complex residential needs for today's people. This article is based on the concept of " renovation and restoration ", combined with practical investigation and research, made a detailed analysis on the renewal of the residential environment of the old residential areas in Hangzhou from sociology, culture, economics and some other aspects, systematically sorted out the complex and multi-dimensional research paths, drew out work frame of "renovation of old residential area". Furthermore, this paper through the analysis and calculation of the Mixed functional indexes such as Space Syntax, Spacematrix and MXI, studied the technical path of the renewal of the old community and put forward the feasible reconstruction strategy.
\end{abstract}

Keywords: Renovation and Restoration, Living Environment, Old Communities, Sociology, Economics, Culture, Compound Path

\section{Introduction}

"Restoration and Repair of the City" refers to an ecological, refined, and sustainable urban renewal method which guides the transformation and development of cities to solve problems, such as insufficient urban development space, poor ecological environment, and lack of social culture in the era of rapid urbanization. [1]. It emphasizes the attention paid to both function and aesthetics, as well as the function to provide design guidelines for the governance of buildings and public spaces in the process of urban development, so as to create a high-quality and sustainable physical environment for urban residents [2]. Over the years, scholars and experts at home and abroad have talked about urban renewal and transformation from different paths. In the west, which is mainly represented by British and American countries, there has been "urban renewal/reconstruction", "urban redevelopment", "urban renewal/renaissance" and "neighborhood renewal"[3]. In China, well-known scholars such as Liangyong $\mathrm{Wu}$, Yisan Ruan, Gengli Zhang, etc, based on the perspective of "organic urban renewal", have made beneficial explorations from various aspects, including "protection and inheritance of culture veins, and harmonious development of society", most of which focus on physical space environment.[4-6]. Since further constraints on urban ecological red line and growth boundary issued in 2014, more scholars such as Baoxing Qiu and Shaoqin Zhuang have begun to introduce advanced foreign concepts such as "sustainability, ecology, and humanity" to conduct more extensive explorations [1]. In the research of scholars' studies and exploration of reality transformation, we can find that under the "new normal", urban renewal has become a main method of urban development in China [7].

With respect to current situations in renewal design of specific areas like old communities, people pay much attention to fundamental renewal of residential building and physical environment, but little attention to synergetic researches from the perspective of sociology, culturology and economics. Living environment renovation in old communities is an integrated research covering different 
disciplines. It requires a compound-path exploration from various dimensions so as to achieve the goal of sustainable and coordinated development.

\section{Existing Problems and Connotation Expansion of Living Environment Renovation to Old Communities}

\subsection{Existing Problems in Living Environment to Old Communities in an Incremental Era}

Based on a study of a series of policies issued by Hangzhou city government, such as the Implementation Plan of Comprehensive Renovation and Upgrading of Old Communities in Hangzhou, the Four-year Action Plan for Comprehensive Renovation and Upgrading of Old Residential Districts in Hangzhou (2019-2022), and the Technical Guide of Comprehensive Renovation and Upgrading of Old Communities in Hangzhou (try out), the author visited and investigated about 10 typical old communities in the first batch of pilot projects published in 2019, including Xingong community in the upper urban area, Zhugan lane, Xiaotianzu Community and Zhizunong community in the lower urban area, Dujia new village in Gongshu District, and Jingtan Community in Jianggan District. Based on the field survey of old residential districts in different regions of Hangzhou, the author distributed questionnaire, conducted field survey and paid visits to local residents in different age, with different occupations and in different communities to collect their opinions on the current situation of old communities and their suggestions for renovation. After collecting data, with the residents' cognition as a variable, the author made systematical analysis and summarized specific problems as follows:

(1) The space place has the problem of single function with poor experience: during the incremental era of the early stage of socialism when the demand for housing and other items is growing rapidly, in the planning of residential areas, people take meeting the largest demand for housing as the design and construction goal, inevitably ignoring the quality of human settlements to some extent; In this way, the public space environment of the residential districts enjoys relatively high floor area ratio, but insufficient open space, which will inevitably lead to problems such as deficiency in green space ecosystem, traffic congestion, and insufficiency of public service facilities, further resulting in environmental problems including rigid space, single function, and placement of vehicles at will; the function of space place needs to be improved.

(2) Lack of community vitality with outstanding isolated problem in living: with the rapid development of urbanization, the functional spatial layout of different areas in Hangzhou has also changed accordingly. The springing up of new residential districts leads to juxtaposed new and old residential areas, which disrupts residents' normal daily life and living habits, further making the scope of social communication be cut off and neighborhood relations become alienated. Gradually, a phenomenon of isolated living appears among people in the same community and people in different communities in the same area. As far as the current social situation is concerned, due to a long-term tendency to material infrastructure construction in the renovation of old communities in Hangzhou, with a negligence of the protection of the social network in the community in which interpersonal relationship is the main body, and the important role of the public space system in the community in the life communication of residents, some communities lacks vitality and has prominent problem of residential isolation.

(3) The loss of urban features with increasingly highly-assimilated designs. Hangzhou, which is known as "heaven on earth", boasts a long history with profound culture contents along the way from "Liangzhu Culture". As one of the most typical spatial areas that can reflect the historical style and cultural characteristics of Hangzhou, old communities are characterized by the regionalism of local life and the diversity of interpersonal communication, standing as an important constituent of urban organization. As a product of the continuity and preservation of history, old communities can reflect not only the quality of local residents' life and living conditions, but also the historical and cultural renovation of a city in the process of spatial development. However, as far as the current situation of the communities is concerned, old communities are deficient in regional characteristics to leave an indelible impression on their residents; There are few public landscape nodes and landmark buildings.

\subsection{The Connotation Expansion of the Environmental Reform of the Old Communities in the Age of Stock}

In recent years, with the development of big data and diversity in cities, the traditional spatial environment model of old communities gradually has difficulty in adapting to the diversified and complex needs of human settlements. To solve various problems brought out by single space function and deficiency in interpersonal communication in traditional old communities of Hangzhou, the residential districts are bound to experience the renovation from a single living mode to a multi-dimensional, ecological and comprehensive one.

With regard to policies, from some opinions of the Central Committee of the CPC and the State Council on further strengthening the management of urban planning and construction issued by the Central Committee in 2016 and the guidance on strengthening the repair of ecological restoration cities officially released by the Ministry of Housing and Urban-rural Development of the People's Republic of China in 2017 , to a new path of urbanization with people as its core put forward on the 18th National Congress of the Communist Party of China, a new path with Chinese characteristics featured with humanism, harmonized development of four 
modernizations, namely industrialization, informatization, urbanization and agricultural modernization, optimized layout, ecological civilization, and cultural heritage, all deeply emphasize the key tasks in "double repair", such as "filling up infrastructure debts, increasing public space, improving travel conditions, transforming old communities, protecting history and culture, and shaping the style of city time" [8], which are in line with the design of optimizing and updating of old residential areas in recent years.

In recent years, Zhejiang province has also put forward various relevant policies, such as the experimental scheme for future community construction in Zhejiang province, and the implementation plan for Hangzhou to implement the construction action of the whole Zhejiang province. Based on various explorative researches on the sharing of overall living space, cultivation of neighborhood emotion, satisfaction of the traffic, and the quality of life in blocks, we found that despite many problems in their internal and external space, old communities, with a strong flavor of life, enjoy a large renovation flexibility and intervention space, thanks to the particularity of their formation. According to the reflection on the previous demolishment and reconstruction, as well as thinking of the severe challenges faced by the communities in their future development and the realistic problem of stock land, the design of the old communities' upgrading needs to break through the material space level to study from the perspective of a compound environmental complex such as the "adaptive renovation" of the composite space, the "cultivation of vitality" in shared space of communities, and the "activation of local cultural capital" of the public space.

\section{Exploration of the Research Path of the Renovation of Old Communities}

As China's cities gradually change from external expansion to connotative development, old urban areas in the stock age have begun to consciously promote the urban upgrading [9]. Based on the analysis of CiteSpace, a literature research map, at present, the research of material renewal is still dominant in China's urban renewal theory without forming a network featured with strong connections; In foreign countries, co-authors around David Harvey, such as Richard Florida and other representatives of non-traditional architectural schools, and some other professional fields are more or less intersected with political economy, sociology and other fields. This indicates that the consensus foundation of foreign countries for urban renewal has changed from the original "material space determinism" to a more realistic or humanistic perspective. Besides, the professional field of urban renewal has expanded from connotation to extension, and has formed a consensus basis featured with clear structure system and relatively intensive internal relations [10].

Based on the above comprehensive discussion on the existing problems in the function, spatial vitality and regional features of the old communities, the author, combining the survey data and the analysis of the satisfaction of the citizens, concludes that the renewal of the old communities in Hangzhou requires not only a simple remedy of the design and planning. That also requires us to rise from the level of material repair to a multi-level and comprehensive discussion and study from perspectives of sociology, culture, and economics to make use of the conclusion of the comprehensive analysis for following renewal design of planning in return. The specific analysis is as follows.

\subsection{Sociological Perspective: The "Adaptive Renovation" of Complex Space and the "Vitality Creating" of Public Space in Communities}

Historical experience shows that the urban renewal with the focus on material environment renovation has destroyed the social fabric of the city due to the lack of attention paid to social problems, thus bringing about many social problems. The old communities, characterized by the dual connotation of sociality and public space, are confronted with complex and diversified issues including public and private space in communities. Our renewal and renovation should, combining the concept of humanism, emphasizes the "adaptive renovation" of complex space in the renovation process, that is, expanding a single dimension of material environment to a multi-dimensional level of society, economy, environment and culture, respecting the wills of residents of different classes, studying the complexity of public participation, and paying attention to the "vitality creating" of public space in communities. We will transform our renewal design from image construction to exploratory research on the mechanism of internal action. For example, general planning of cities in Shenzhen City (2010-2020) takes the lead to propose that the spatial development should be changed from "incremental expansion" to "stock optimization". The plan focuses on key contents under the new development values that emphasize "overall", "endogenous", "integrated" and "culture-oriented" development, which is very worthy of our reference [11].

With detailed interpretation of "Notice of declaration work on the construction of pilot future communities in Zhejiang Province issued by Zhejiang Provincial Development and Reform Commission in 2019", we can think about in the construction of future community neighborhood, how to combine the scene with various designs such as system architecture, space carriers, visual design, scale standards and mechanism guarantee to study the modification scheme and implementation methods in community open ans sharing of neighborhood space. From that, it can be seen that the renovation of old communities is not a single entity, but a complex sociological problem. According to the above analysis, the author, taking into consideration various needs of residents in society, economy, culture and life, draws the SWOT analysis chart (Figure 1) to guide the subsequent planning and design. 


\section{+ SWOT}
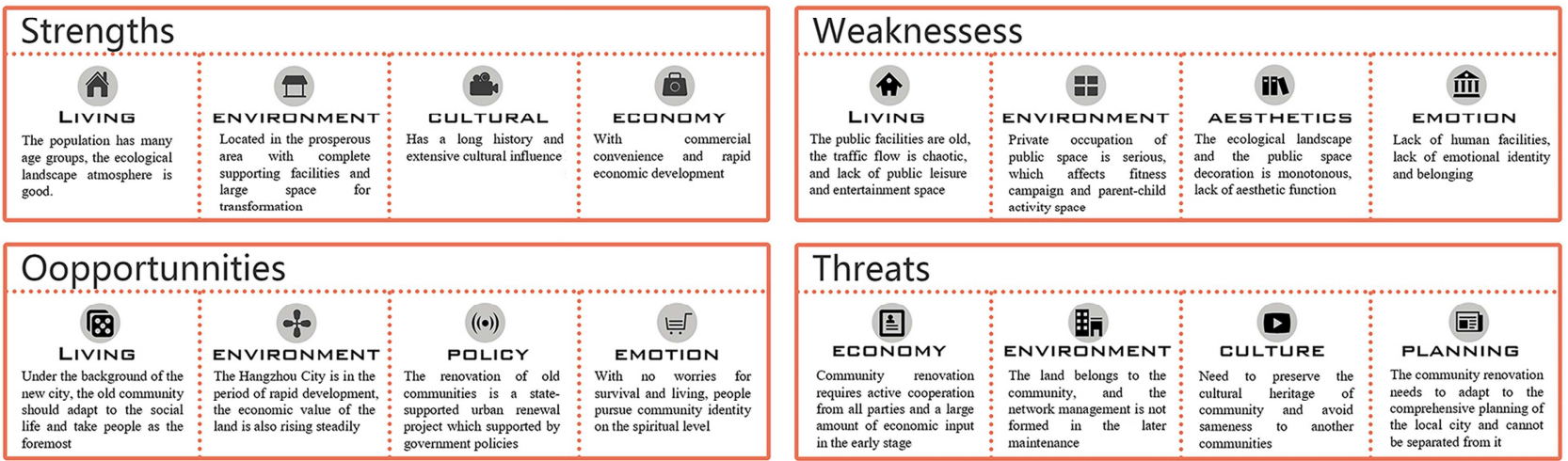

Figure 1. SWOT Analysis Chart of Current Situation of Residential Area.

\subsection{From the Perspective of Culturology: Improve Cultural Cognition and Perceptual Experience Under the Background of Shared Communities}

As for the importance of culture, Pierre Bourdieu, a French sociologist, pointed out that in the contemporary society, culture has penetrated into all fields and overtaken traditional factors such as politics and economy to take the top spot in social life. Based on the concept of cultural capital, urban renewal can be understood as a process of continuously activating, creating and accumulating urban cultural capital [12]. From the perspective of culturology, the old communities, as an important carrier of urban spatial structure, not only records the urban spatial pattern and construction mode, but also embodies various connotation of the relationship between city and society, as well as between city and humanity. Under the planning background of "building a city with culture", only when the spatial environment around the old communities become the living place and space for daily culture to stimulate people to develop the depth and thickness of culture here [13] can old communities in Hangzhou really arouse people's resonance and sense of belonging to their living space, and make them essentially different from that of other cities. To allow residents to perceive due cultural atmosphere, historical characteristics and nostalgia from the city and communities, it is of important significance to implant cultural elements synchronously tin the process of renovation.

According to the investigation and visits, the author found the human settlements of old communities in different areas of Hangzhou is generally simple, the design of public facilities is not systematic enough, and the cultural inheritance has not been paid enough attention to. The author took Dujia new village in Gongshu District of Hangzhou as the research case and drew up the following design of its public facilities. Considering that the new village, which is close to the Beijing-Hangzhou Grand Canal, carries the spatial meaning of "canal culture" and water towns, the author, by combing the elements of "canal culture", designed boat--shaped bus stations outside residential areas, and boat-shaped rest seats, containers for green plants, street lamps, and landmarks inside residential areas (Figure 2). Meanwhile, taking into consideration of the aesthetic needs of modern people, the author also made use of stainless steel and other materials to design relatively fashionable sailboat-shaped bus platforms and rest seats (Figure 3). Both of the above schemes, by making use of the cultural element of "boat" and the architectural elements in horse-like wall in water towns to modify the infrastructure, makes it possible for "water towns" and "canal culture" be better inherited around the residential areas of Hangzhou.

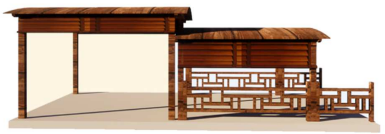

Bus station

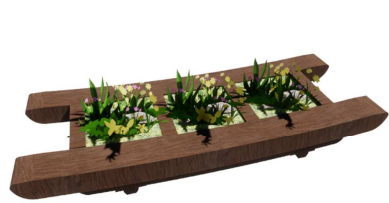

Landscape tree pond

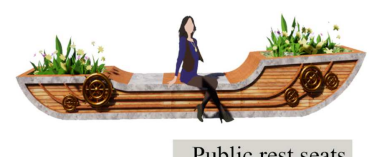

Public rest seats

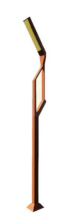

Road Lamp

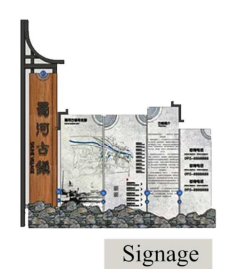

Figure 2. Public Facility Design of "Canal Culture" (1).

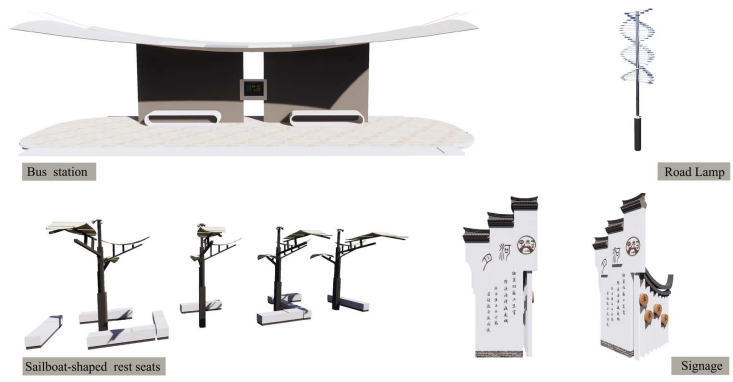

Figure 3. Public Facility Design of "Canal Culture" (2). 


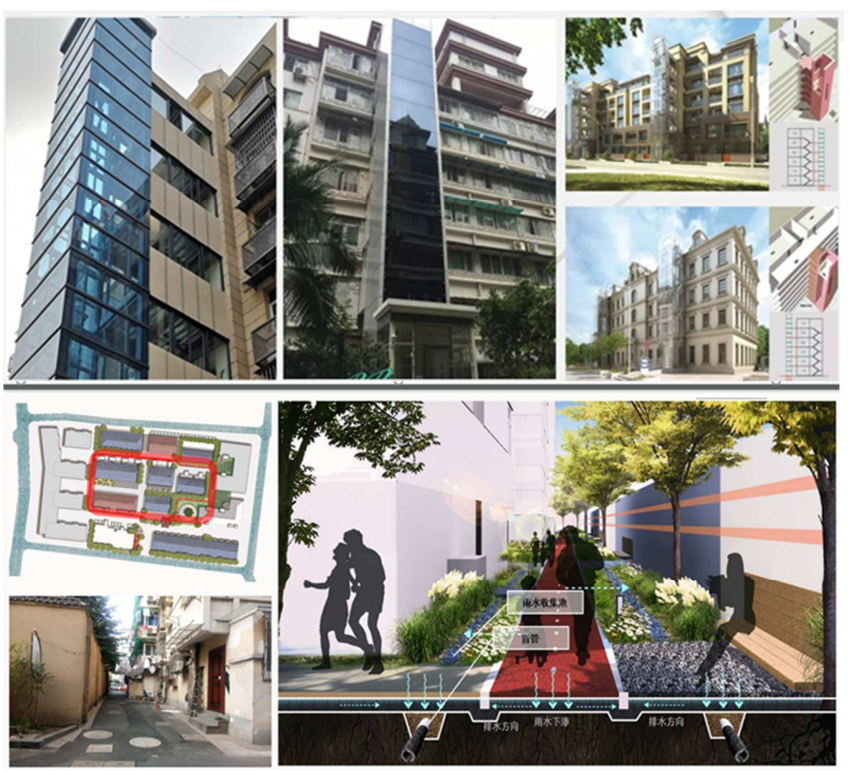

Figure 4. Schematic diagram before and after the Gradual Renovation of Standardized Regions Plan of Old Community of Nanbanxiang.

\subsection{From The Perspective of Economics: The Combination of "Standardized Regions" and "Organic Renewal ", a Study of the Universality and Individuality}

From the perspective of economics, it is of greater difficulty in transforming old communities based on the "reuse of stock land" than building new ones. That requires us to do scientific and rational renovation based on the integrated analysis of the current situation. Taking economics into consideration, we, after conducting deep study on the current situation of human settlements in old communities in Hangzhou to systematically classify their public space environment, creatively put forward the renewal ideas that combine "gradual renovation of standardized regions" and "organic renewal of personalized space", which can coordinate both universal and individual needs of the residential areas in the process of renewal and renovation.

(1) "gradual renovation of standardized regions". First of all, residential areas, as a public space with systematic planning and design, must have something in common in their designs. In terms of the author's investigation and visits to old communities, after data analysis is carried out based on the "material" level, renovation of many items can be updated according to the standard dimension. For example, in the old community of Nanbanxiang in the above urban area, its building facade, outdoor cabinets, canopies, catch basin covers can be upgraded in such a way (Figure 4). Besides, considering that these kinds of renovation often have common standards with that of other old residential areas in the same period, this method can be analogized to the renovation of other old communities.

In accordance with the above common problems, we put forward the concept of "gradual renovation of standardized regions". Under guidance of the concept, we plan to start from the small parts of the existing visible space that need to be repaired, such as the reconstruction of the old residential building facade, the road system, greening system, and the infrastructure supporting facilities to carry out standardized and systematic renovation, so as to save human, material and financial resources to the maximum extent. What's more, through the "gradual renovation of standardized residential landscape environment", we plan to achieve renovation and management program with innovative mechanism and long-term management; we hope to build a systematic management mechanism which is featured with"one-time renovation and long-term maintenance".

(2) "organic renewal of personalized space". Interpreting from the connotation of urban double repair, old communities achieve space's regeneration through ecological restoration; it is a personalized behavior that targets at a specific region. That requires us to, in the process of renovation, reexamine the development history and current situation of the city to the street, and the community, to analyze the historical changes of the spatial environment and differences among residents, so as to select upgrading methods in a reasonable and discreet way. That is why we put forward the concept of "organic renewal of personalized space" in the renewal of landscape nodes, infrastructure and public space in different communities. Inline with the requirements of "protecting the foundation, promoting the upgrading, expanding the space and increasing the facilities", we optimize the utilization of space resources in and around residential areas, make clear the contents and basic requirements of personalized space renovation, and strengthen the guidance of designs, so as to achieve "every single residential area has a unique scheme "(Figure 5). Take the special reconstruction of old communities such as Zhugan Lane in upper urban area, Hangzhou for example. In order to solve social problems such as low participation, weak sense of belonging, and lack of community construction of the community, we, by carefully considering the spatial layout, residents, social needs and other elements, carry out 
"customized" expansion design with certain targets and plans to the nodes of landscape for public communication and multi functions of public facilities of the residential area. In addition, targeting at different groups, we can set out personalized innovation schemes, such as that of the renovation of Xiaotianzhu community. After practical investigation, we, with focus on the residents most of whom are the aged, unify the color and style of the greening landscape facilities in public rest areas, combined with elements in traditional Chinese buildings, to design a small-scale garden of ancient charm with the flavor of culture in the Southern Song Dynasty, thus creating a comfortable and livable garden-style community full of Zen and ancient charm of water towns (Figure 6).
As far as the practical significance is concerned, the "organic renewal of personalized space" not only maintains the basic functions of communities and expands public space and supporting services, but also activates the living space of space experience and living experience of old communities, which makes it possible to ensure the lasting of neighborhood space with close communication [14]. At the same time, it is necessary for us to conduct the renewal and renovation of each residential area in stages and with emphasis, so as to reserve time and space for reflection for the "urban double repair", which is important to strike an efficient balance between"individuality and universality" and realize the multi-dimensional development of old communities.

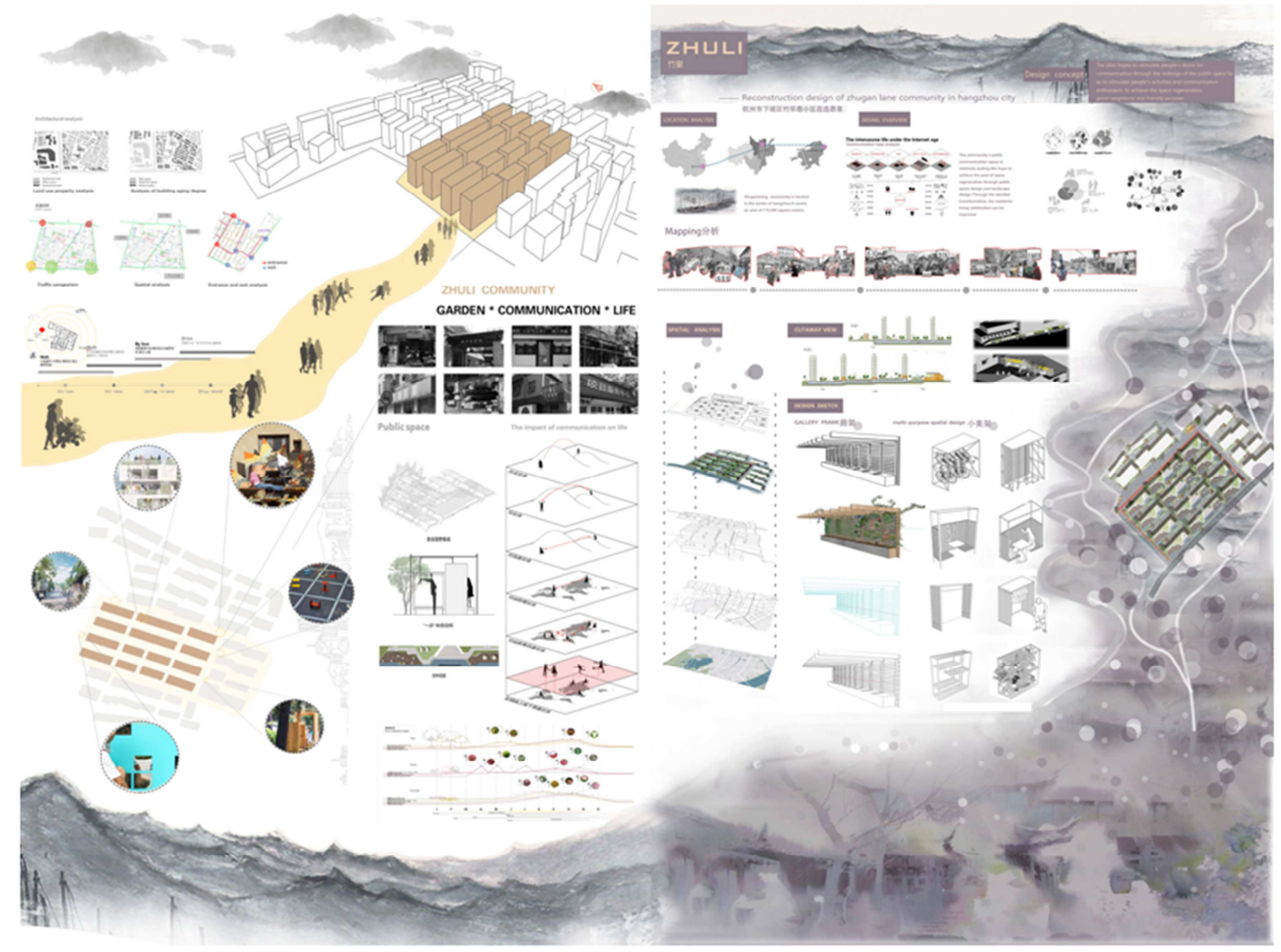

Figure 5. Regional Renovation Plan of Old Community of Zhuganxiang.
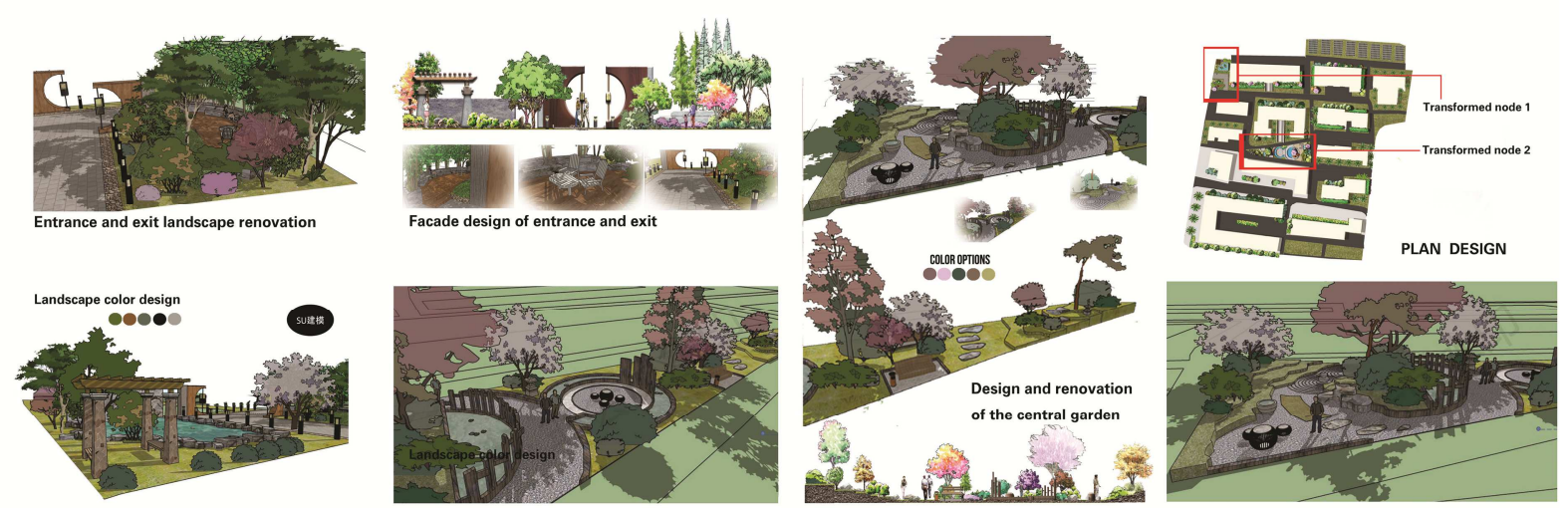

Figure 6. Public Space Renovation Plan of Old Community of Zhuganxiang. 


\section{Analysis of Work Frame and the Technical Path}

From the macro perspective, our renewal design needs to combine the harmonious and inclusive social concept, the pluralistic and interconnected cultural concept and the dialectical and rational economic concept to be coordinated and renewed through the above researches on the reconstruction path of the demands and needs of the old communities. Based on this, the writer combines big data, Spatial Syntax, urban morphology and other technical path analysis methods to draw the work frame and analysis diagram of technical path, so as to provide reference for the following renovation.

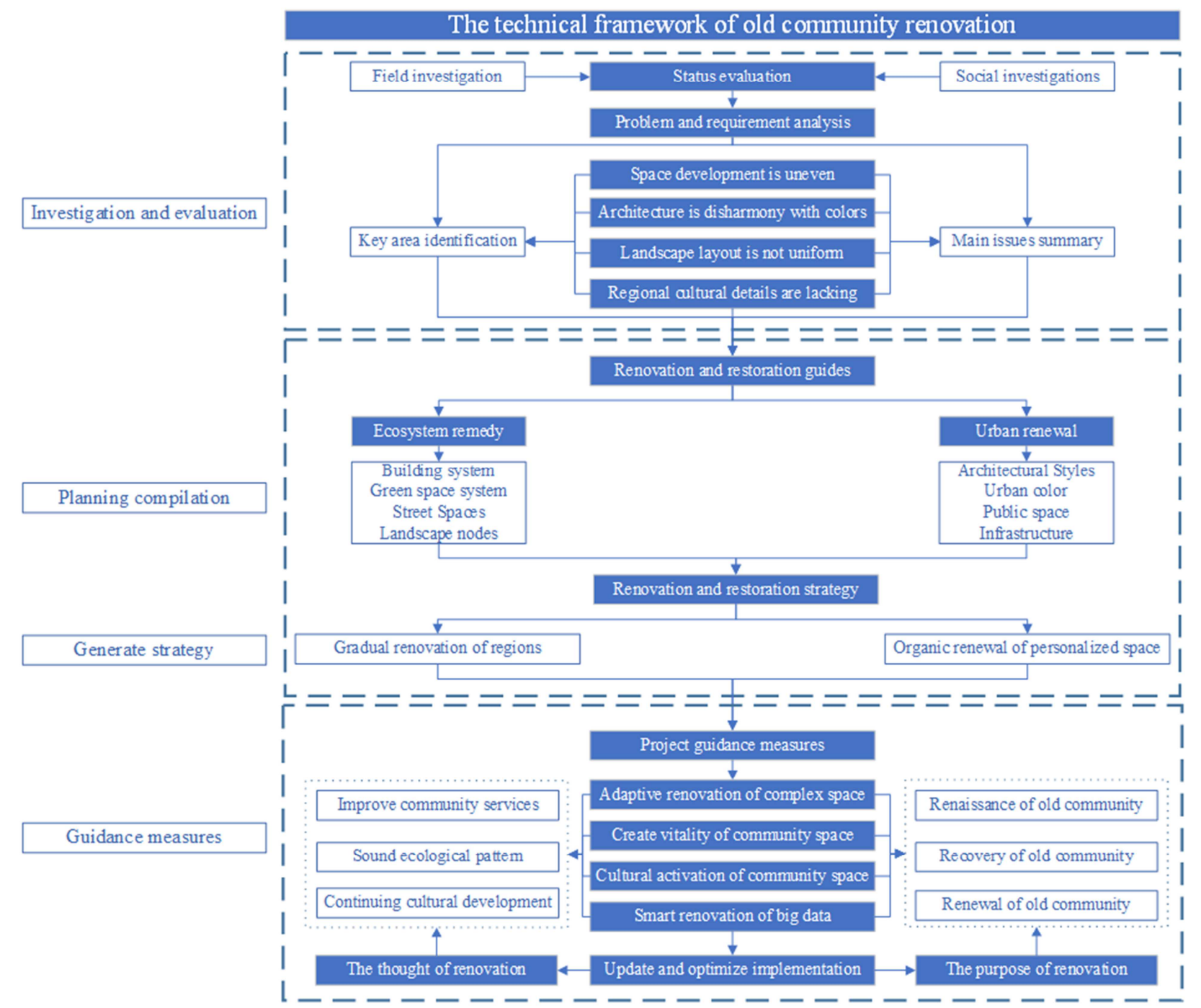

Figure 7. Technical Block Diagram of "Renovation and Restoration" to Old Communities.

\subsection{Analysis on the Work Frame of "Renovation of Old Residential Area"}

Since urban renewal involves many complex interests, the success of renewal and reconstruction need to be ensured by the establishment of a truly effective urban renewal governance system, an open and inclusive decision-making mechanism, and a cooperative and coordinated implementation process [15]. Therefore, the writer refers to various relevant policies, such as "The Experimental Scheme for Future Community Construction in Zhejiang Province". Based on the principle of "overall control from macro level, systematic combine from mesoscopic level, detailed implementation from micro level", the writer draws a "Frame Diagram of Renovation And Restoration " (Figure 8) in details from the survey to the implemented works at all stages, puts forward some problems that need to be solved in "urban restoration" as well as "ecological restoration", and thinks about the existing problems from point to surface, so as to provide an effective framework for the sustainable development of communities to guide the following work.

\subsection{Analysis of Compound Technology Path}

In the big data age, our urban design has transited to the stage of quantitative and scientific rational analysis combined 
with big data such as urban morphology and Spatial Syntax rather than the previous traditional era of space construction, which only relies on the intuition and experience of designers themselves. Therefore, on the basis of surveys, the writer combines a series of quantitative urban morphology analysis tools such as Spatial Syntax with the planning and design theory of communities to analyze the patterns of spatial vitality of old communities in Hangzhou, so as to make quantitative analysis on the construction of spatial vitality in multiple stages of residential renewal designs.

Based on the above analysis of the problems of the old communities, we put forward to study the technological path of renewal and reconstruction through the way of experimental design, which is roughly divided into two parts. On the one hand, based on GIS, we combine the qualitative traditional theory of morphology with the new quantitative method (Space Syntax, Spacematrix and MXI) to analyse the urban morphological characteristics of the spatial vitality of the communities. On the other hand, we test effectiveness of
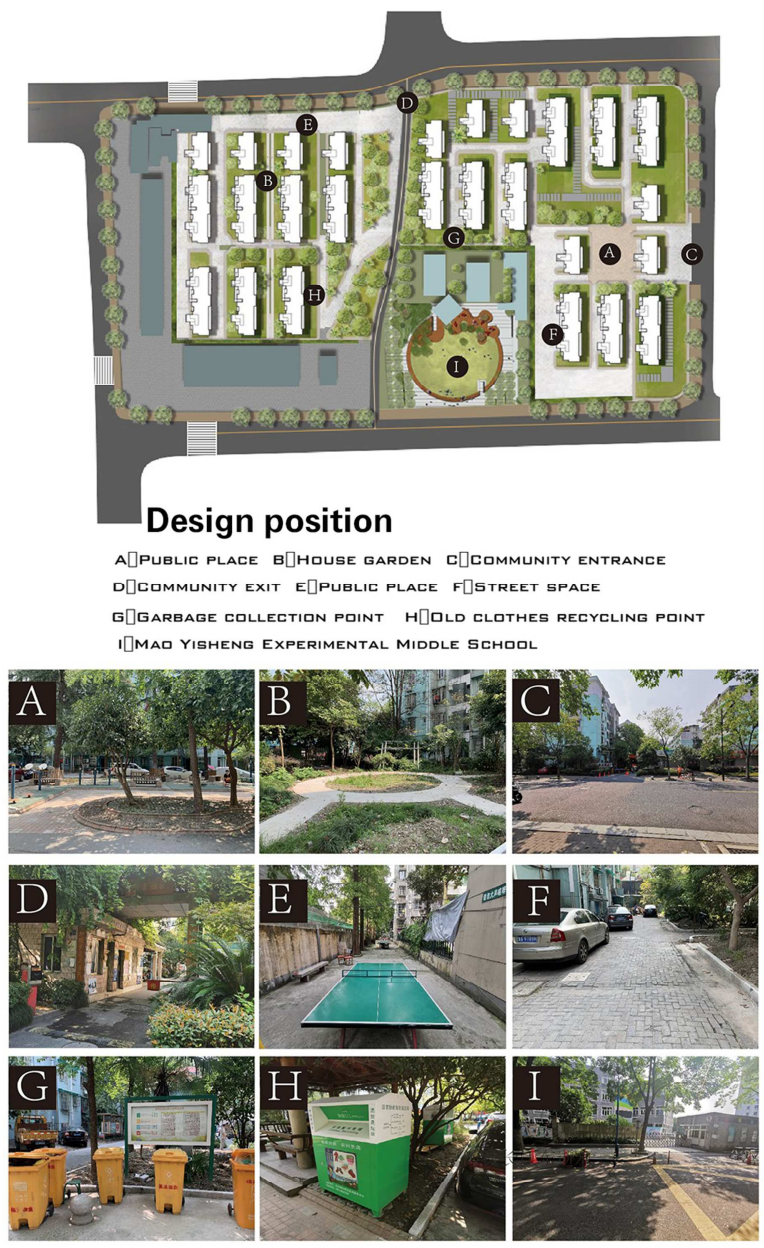

the above analysis from the perspective of residents' selective activity intensity. Then, we will take Jingtan Community in Jianggan District as an example to make the technical demonstration.

First, we use Space Syntax to make the spatial connection relationship abstraction and fabric analysis of the traffic system of Jingtan Community, and this way can reflect the traffic accessibility of the communities to a certain extent through the data image. Second, we can effectively define the spatial form layout of the communities through the Spacematrix that based on the data analysis of plot ratio, construction intensity and floor height. Simply speaking, the way defines the spatial characteristics of the community by analyzing the intensity of residents' activities. Third, based on the calculation of MXI (Mixed-use Index), we can define the functional mixing degree of residential area by the ratio of building area of three main functions: living, public space (work) and infrastructure (Figure 9).
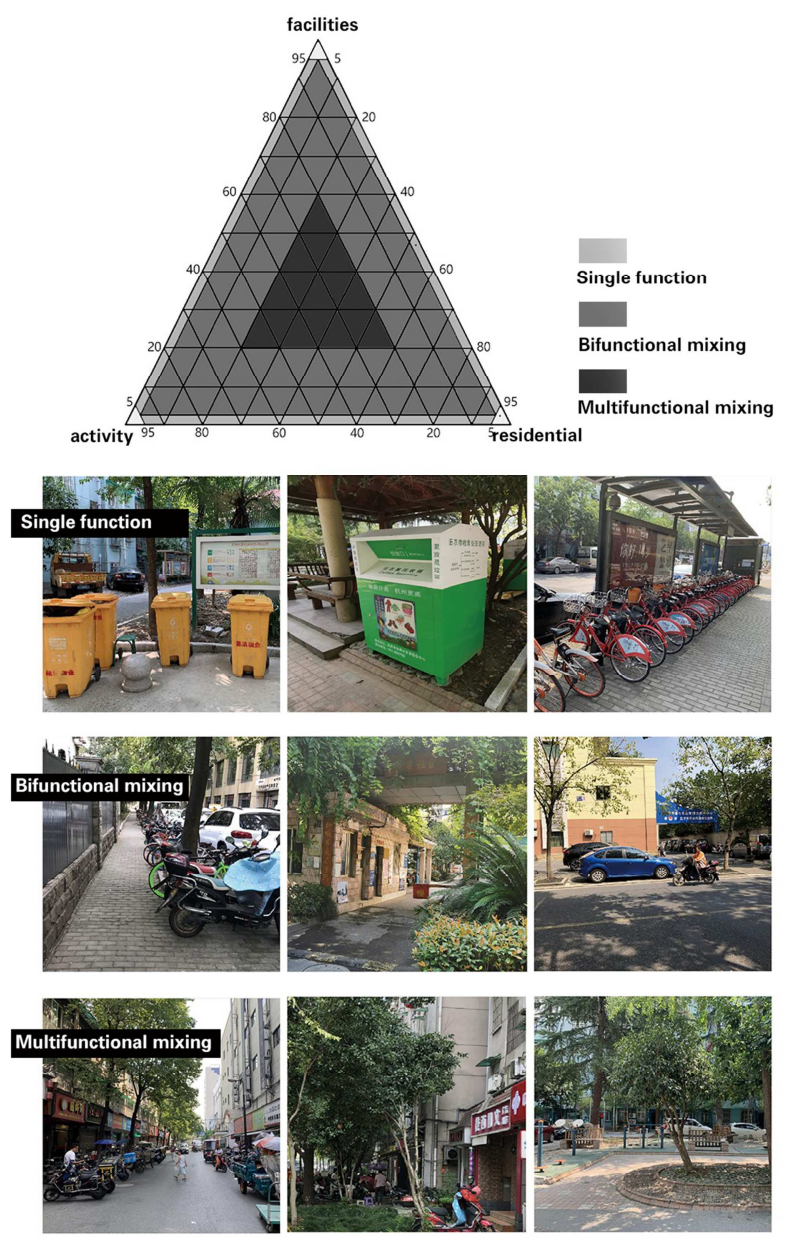

Figure 8. Schematic Diagram Based on Spacematrix and MXI of Jingtan Community.

Based on the above analysis, according to the calculation of MXI analysis (formula: MXI $=$ living $(\%) /$ working $(\%)$ facilities $(\%)$ ), we can predict the analysis chart of functional mixing degree of residential environment space, and the chart can accurately reflect the space situation of Jingtan Community to guide the following reconstruction. 


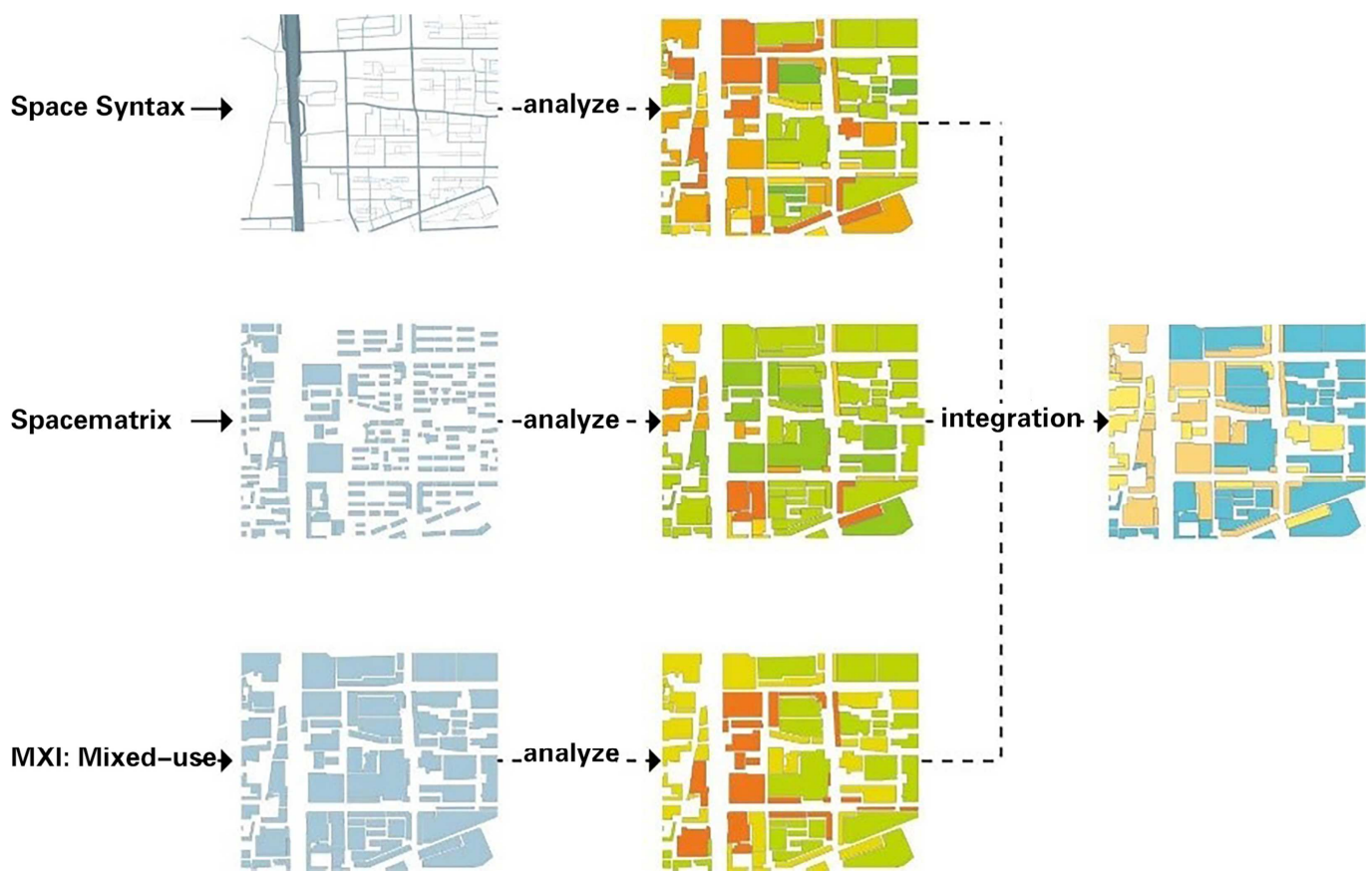

Figure 9. Schematic Diagram of Space Syntax, Spacematrix and MXI Analysis of Jingtan Community.

Through the data analysis of the three methods of Space Syntax, Spacematrix and MXI of the areas of communities (Figure 10), we can respectively analyse and describe the accessibility of the residential space, the intensity of the plot construction and the compound conditions of architectural form and the plot function [16] of the Jingtan Community. According to the panel points of data-based and scientific analysis and reconstruction that from the perspective of factors such as crowd activity, we study the renewal design of the spatial form of the communities, such as the traffic, building height, public landscapes, public activity space layout, so as to collect the basic data of the renewal design of the old communities in different regions.

The above research shows that the cultivation of urban space vitality is not a relatively vague concept that lacks of practical measurement. With the purpose of ensuring a better effect of the design and renovation, we can guide the future update design through the analysis of data to provide a solid foundation for building a good traffic accessibility of the community, a suitable layout of the building space, and a certain level of mixing degree of public space functions. Meanwhile, we can test the effectiveness of the design and reconstruction from the data analysis of non spatial elements such as the strength of residents' selective activities after the renovation. We can know that these quantitative data recorded on the map can help to implement the urban design from the original "traditional intentional design" to "multi-dimensional compound design research that "based on big data" as the reference for our updated design, a new perspective for the construction of urban space vitality.

\section{Conclusions}

The reconstruction of the community is a process of sustainable regeneration and activation, which is conductive to the comprehensive improvements full of recessiveness and dominance in urban development context, beautification of urban environment, improvement of living conditions of residents, etc. In the process of reconstruction, we should fully combine the internal artistic conception of social and cultural attributes to reconstruct and integrate the external space areas, and make a comprehensive consider of the multi level renovation of economic benefits. As the above step by step analysis, we should further clarify that the basic responsibility of urban renewal is to cultivate the living environment, and the expanded responsibility is to coordinate the reopening of the city [17]. We should reconstruct our familiar places, make our communities have the ability to face the future, develop a sustainable and resilient future community, so that the regeneration and activation of settlements can form a virtuous circle and interaction under the premise of "stock planning" and the requirements of improving urban services and the ecological pattern and highlighting the cultural background.

\section{Acknowledgements}

This work is supported by 2020 Philosophy and Social Science Planning Project Youth Project of Zhejiang Province (NO.: 20NDQN323YB).

\section{References}

[1] LEI Weiqun, XU Shan, ZHOU Yong, WEI Bo, ZHU Liying (2018). Theoretical Explanation and Practical Exploration of Urban Betterment and Ecological Restoration, 11: 32-36.

[2] Lang Wei, Li Xun, Chen Tingting (2017). Evaluating Sustainable Urban Renewal Approach in Hong Kong from a Social Perspective, 10: 1-9. 
[3] YANG Jianbo, LI Fan, HUANG Wei (2015). Evolution, Mechanism and Restriction of Utilizing the Conception of Urban Renewal for Famous Historical and Cultural Citie: A Case Study of the Transformation of "the Three Olds" in "Lingnan Tiandi" of Foshan. Tropical Geography, 3 (35): 170-177.

[4] YANG Jianqiang, WU Mingwei (1999). Modern Urban Renewal. Nanjing: Southeast University Press.

[5] ZHANG Gengli (2004). Towards Three-way Partnership in Urban Regeneration: The Western Experience and Implications to Chinese Cities. Urban Studies, (4): 26-32.

[6] RUAN Yisan (2003). A Documentary on City Protection. Beijing: China Architecture \& Building Press.

[7] ZHANG Lei (2015). The Modes of Urban Redevelopment in New Normal: Diversification and Transformation. Urban Development Studies, 12 (22): 57-62.

[8] JING Bo, DING Yuyuan and HAN Ting (2019). Accurate, Humanism, Traditional: Exploration on the Orientation of "Urban Renovation and Ecological Restoration" Planning of Historic Urban Area: A Case of Xi'an Old Town. Modern Urban Research, 000 (004): 112-120.

[9] YAN Huilv, WANG Hui (2019). An Adaptive Approach of Quality Improvement for Old Buildings Under Repair and Renocation Background, Hami City. Planners, 5 (35): 53-59.

[10] ZHU Yijia, LI Hui and WANG Wei (2015). Characteristics and Trend of Urban Renewal Researches. Urban Problems, 9 (242): 30-35.
[11] ZHOU Tingting, XIONG Yin (2013). Inventory Optimization of Land Use and Urban Renewal. Planners, 2 (29): 36-40.

[12] HUANG Yi, WU Changfu and XIE Zhenyu (2015). Stimulating Local Cultural Capital in Urban Renewal: The Case of Urban Renewal Program and Planning of Jieguan Lane, Tengzhou. Urban Planning Forum, 2 (222): 110-118.

[13] DING Zhicai, CAI Sihan (2017). Rejuvenation of Residential Area and Inheritance of Culture---An Analysis of the Development of Quanzhou West from the Perspective of Historical Streets Redevelopment Experience in Japan. Journal of Ocean University of China, 5: 85-90.

[14] YANG Junyan, SHI Yi (2015). A Study of the Mode of Preserving Historic Districts Based on 'Micro-Community'-From a Socio-Spatial Perspective. Architectural Journal, 02: 119-124.

[15] LI Angxuan (2019). The Enlightenment of the Process of Urban Renewal in Britain to China. Special Zone Economy, 3 (362): 101-103.

[16] YE Yu, ZHUANG Yu, ZHANG Lingzhu and Akkelies van Nes (2016). Designing Urban Spatial Vitality from Morphological Perspective-A Study Based on Quantified Urban Morphology and Acticities' Testing. Urban Planning International, 1 (31): 26-33.

[17] WANG Shifu, SHEN Shuangting (2015). From "Three-Old" Reconstruction to Urban Renewal-Thinking around the Newly-established Uurban Renewal Bureau in Guangzhou. Urban Planning Forum, 3 (223): 22-27. 